\title{
Separation of Metallic and Semiconducting Single-Walled Carbon Nanotubes Through Fluorous Chemistry
}

\author{
Sandeep Ghosh ${ }^{1,2}$ and C. N. R. Rao ${ }^{1,2}(\bowtie)$ \\ ${ }^{1}$ Chemistry and Physics of Materials Unit, DST unit on Nanoscience and CSIR Centre of Excellence in Chemistry, Jawaharlal Nehru \\ Centre for Advanced Scientific Research, Jakkur P.O., Bangalore-560064, India \\ ${ }^{2}$ Solid State and Structural Chemistry Unit, Indian Institute of Science, Bangalore-560012, India \\ Received: 4 November 2008/Revised: 18 December 2008/Accepted: 18 December 2008 \\ CTsinghua University Press and Springer-Verlag 2009. This article is published with open access at Springerlink.com
}

\begin{abstract}
Separation of metallic from semiconducting single-walled carbon nanotubes has been a major challenge for some time and some previous efforts have resulted in partial success. We have accomplished the separation effectively by employing fluorous chemistry wherein the diazonium salt of 4-heptadecafluorooc tylaniline selectively reacts with the metallic nanotubes present in the mixture of nanotubes. The resulting fluoroderivative was extracted in perfluorohexane leaving the semiconducting nanotubes in the aqueous layer. The products have been characterized by both Raman and electronic absorption spectroscopy. The method avoids the cumbersome centrifugation step required by some other procedures.
\end{abstract}

\section{KEYWORDS}

Carbon nanotubes, metallic, semiconducting, fluorous, separation

Single-walled carbon nanotubes (SWNTs) possess many remarkable and useful properties [1, 2], but research and development in this area suffers from a major limitation because of the absence of a foolproof and easy method to separate metallic and semiconducting nanotubes [3]. Such separation is essential for many of the applications which are specific to one or the other type of SWNTs [48]. SWNTs prepared by the arc discharge or other methods generally contain around $\sim 33 \%$ of metallic species. There are several reports in the literature describing methods of separation of SWNTs. These methods can be grouped into two main classes. The first involves sidewall functionalization by reagents such as dichlorocarbene, diazonium salts, fluorine or atomic hydrogen which transforms the electronic structure from metallic to semiconducting [9-13]. The underlying difficulty with such methods is that too much functionalization leads to the disruption of the electronic structure of the SWNTs. The other approach involves selective collection of either metallic or semiconducting SWNTs through AC dielectrophoresis [14], selective flocculation assisted by octadecylamine [15] or porphyrins [16], selective adsorption of $\mathrm{Br}_{2}$ [17], density gradient induced centrifugation [18], and other methods [19]. Despite all these efforts, in most cases the degree of separation achieved is not sufficient for useful applications. Furthermore, most of these methods make use of high-speed centrifugation which is a cumbersome

Address correspondence to cnrrao@jncasr.ac.in 
process and does not also universally ensure complete separation [20]. In this contribution, we describe how metallic nanotubes can be effectively separated using a novel chemical approach viz. fluorous chemistry $[20,21]$ involving the reaction with the diazonium salt of 4-heptadecafluorooctylaniline. Water-soluble diazonium salts are known to react with carbon nanotubes [22-25], and the diazonium salt, in the course of forming a covalent aryl bond, extracts electrons from the nanotubes and provides high chemoselectivity towards metallic nanotubes. The method described here involves reaction of the SWNTs with the diazonium salt and extraction of the nanotubes so functionalized by the fluorous tag using a fluorous hydrocarbon. The method completely avoids centrifugation in the course of the separation. It may be noted in this connection that fluorous chemistry is one of the best methods available today for purification, with nearly 100\% efficiency, as it involves merely attaching a fluorous tag to the substrate and then extracting this tagged moiety into a fluorous solvent.

In order to estimate the relative proportions of the metallic and semiconducting nanotubes, we have employed both electronic absorption spectroscopy and Raman spectroscopy. In the electronic absorption spectrum of the as-prepared and purified arc discharge SWNTs, a band centered around $720 \mathrm{~nm}$ arises from the metallic nanotubes $\left(\mathrm{M}_{11}\right)$ while bands around $1030 \mathrm{~nm}\left(\mathrm{~S}_{22}\right)$ and $1850 \mathrm{~nm}\left(\mathrm{~S}_{11}\right)$ are from the semiconducting ones [26]. The G-band in the Raman spectrum of the SWNTs consists of components due to the semiconducting and metallic species [27 -29]. The frequency and linewidth of the band are sensitive to electron or hole doping, and provide a sensitive means of studying the electronic structure of the SWNTs $[30,31]$. The bands from the metallic components in the G-band are centered at 1540 and $1585 \mathrm{~cm}^{-1}$ and bands from the semiconducting components at 1555 and $1588 \mathrm{~cm}^{-1}$. We have also employed the Raman bands due to the radial breathing modes (RBM) to monitor the changes in the proportion of the metallic nanotubes.

The experimental procedure employed by us is as follows. SWNTs, prepared by the arc discharge method, were purified by successive acid and hydrogen treatment [32]. They were characterized by field emission scanning electron microscopy (FESEM), transmission electron microscopy (TEM), electronic absorption spectroscopy, and Raman spectroscopy. A stable dispersion of these tubes was prepared by ultrasonication with a $1 \%$ aqueous solution of sodium dodecylsulphate (SDS) and subsequent centrifugation to produce a suspension containing singly suspended SWNTs which has a typical mass concentration of 20-25 mg/L [33]. The diazonium salt of the fluorous aniline 4-heptadecafluorooctylaniline (from SigmaAldrich Co.) was prepared following the standard procedure [34], wherein the fluorous aniline was dissolved in dilute hydrochloric acid $(1 \mathrm{~mol} / \mathrm{L})$ and cooled to $4{ }^{\circ} \mathrm{C}$. A cooled aqueous solution of sodium nitrite was added slowly over a period of $2 \mathrm{~h}$ under stirring, resulting in the formation of the diazonium salt solution of the fluorous aniline. This solution $(1.2 \mathrm{~mL}, 51 \mu \mathrm{mol} / \mathrm{L})$ was then reacted with the SDSsuspended SWNTs $(5 \mathrm{~mL})$ by the slow addition to the suspension of the nanotubes under constant stirring over a period of $6 \mathrm{~h}$. This ensured the selective reaction of the diazonium salt with the metallic SWNTs. The reaction mixture was then stirred with the fluorous hydrocarbon perfluorohexane for $24 \mathrm{~h}$ to transfer the fluorous-tagged metallic SWNTs into the fluorous phase [20]. The residue in the aqueous layer, as well as the fluorous extract (both before and after combustion of the organic part), were examined by electronic absorption spectroscopy and Raman spectroscopy. The diazonium functionality from the fluorous extract was removed by heating and the resulting product was characterized. The residue left after diazotization was similarly heated to ensure that no functionalized SWNTs remained in order to avoid errors in the spectroscopic assignments. In this connection, it should be noted that in the absence of the diazonium reaction, both the metallic and semiconducting SWNTs do not dissolve in any solvent, particularly in fluorous hydrocarbons such as perfluorohexane which is highly non-polar with a very low refractive index. Some of the SWNTS (the metallic ones) could be transferred to the fluorous solvent only after reaction with fluorous diazonium compound.

An FESEM image of the purified SWNTs is 


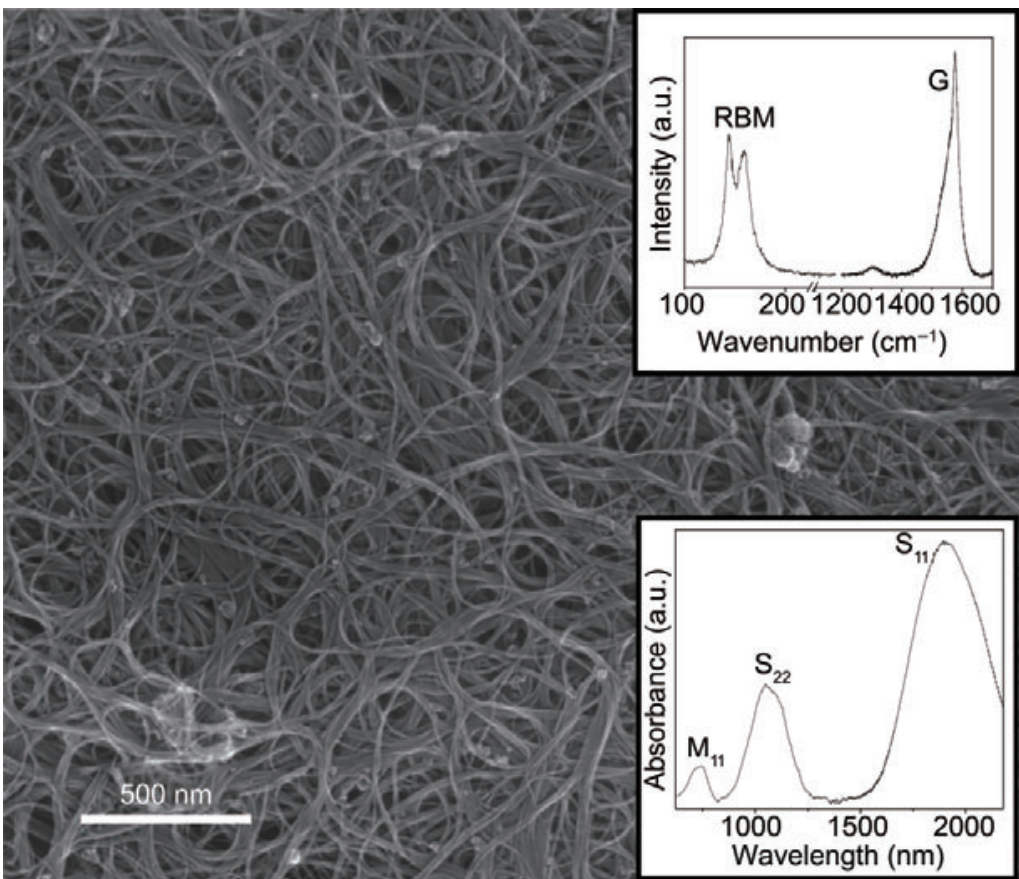

Figure 1 FESEM image of purified SWNTs. Upper inset shows the Raman spectrum of the SWNTs recorded using $632 \mathrm{~nm}$ laser excitation. The lower inset shows the electronic absorption spectrum of the SWNTs

shown in Fig. 1. The upper inset shows the Raman spectrum of the as-prepared purified nanotubes, recorded at $632 \mathrm{~nm}$ laser excitation, where the RBM and the $G$ bands are indicated $[27,28]$. Based on the work of Kataura et al. [26], we assign the Raman bands centered at around 145 and $165 \mathrm{~cm}^{-1}$ as due to semiconducting and metallic SWNTs, respectively. There are reports in the literature of RBM bands at $195-225 \mathrm{~cm}^{-1}$ for certain samples of SWNTs [35], but these refer to SWNTs produced by the HiPco (High Pressure gas-phase decomposition of $\mathrm{CO}$ ) process, which have different diameters from the nanotubes produced by the arc discharge method employed by us. According to Kataura et al. [26], the RBM transitions are dependent on the SWNT diameter (the energy of the transition varies inversely with the tube diameter) and hence consequently on the process by which they are prepared. The positions of the RBM bands found by us are in agreement with the literature reports [36-39] of Raman spectra of arc discharge SWNTs prepared using a $\mathrm{Ni}^{-} \mathrm{Y}$ catalyst. The electronic absorption spectrum of the nanotubes (recorded by depositing the sample on a quartz slide) used by us is shown in the lower inset of Fig. 1. The band centered around $720 \mathrm{~nm}$ corresponds to the metallic nanotubes $\left(\mathrm{M}_{11}\right)$ while the bands around $1030 \mathrm{~nm}\left(\mathrm{~S}_{22}\right)$ and $1850 \mathrm{~nm}\left(\mathrm{~S}_{11}\right)$ are due to the semiconducting nanotubes [26].

In order to see the changes in the optical transitions of the SWNTs induced by the diazotization reaction and subsequent processes, we monitored the electronic absorption spectra at various stages. Figure 2 shows the changes in the optical absorption spectra on reaction with the diazonium salt. The spectra were recorded only up to $1300 \mathrm{~nm}$ as beyond that limit there is interference from water (which is the reaction solvent). The spectrum of the SWNT suspension after the completion of the diazotization reaction is devoid of the metallic band $\mathrm{M}_{11}$ centered around 720 $\mathrm{nm}$ originally present in the spectrum of the suspension of the starting SWNTs. The disappearance of this transition results from the presence of the diazonium functionality. The $S_{22}$ band at $1030 \mathrm{~nm}$, on the other hand, remains almost unchanged. These spectra show that the methodology employed by us is efficient in selectively tagging the metallic SWNTs.

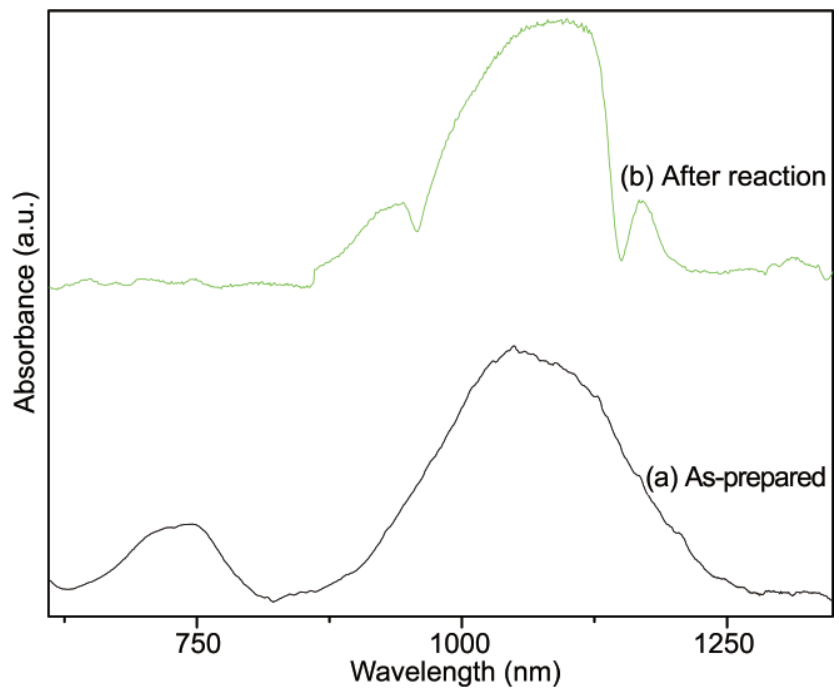

Figure 2 Electronic absorption spectra of the SWNT samples during the course of the reaction of the diazonium salt: (a) as-prepared pure SWNTs in aqueous suspension; (b) aqueous suspension of SWNTs after complete addition of the diazonium salt showing the disappearance of the $M_{11}$ transitions 
In Fig. 3, we have shown the optical absorption spectra of the fluorous extract before and after annealing at $600{ }^{\circ} \mathrm{C}$ and the spectrum of the asprepared purified SWNTs for comparison. The spectra were recorded using solid samples deposited on quartz slides and subsequently washed with copious amounts of water. It is evident from the figure that our method essentially yields nearly pure metallic SWNTs in high yields as the metallic transitions, which disappeared in the fluorous extract, reappeared on heating at $600{ }^{\circ} \mathrm{C}$ which destroys the diazonium functionality. This demonstrates that the fluorous extract consists almost entirely of metallic SWNTs. This evidence for defunctionalization on heating the diazotized SWNTs is further corroborated by the Raman spectra of the fluorous extract (before and after annealing at $600{ }^{\circ} \mathrm{C}$ ) as can be seen in Fig. 4. The disorder band (D-band) in the spectrum of the fluorous extract has high intensity before annealing due to the functionalization by the diazonium salt. After heating the fluorous extract at $600{ }^{\circ} \mathrm{C}$, however, its spectrum exhibits a low intensity of the disorder band which is comparable to that of the starting material. This shows that we are able to remove the diazonium functionality from the fluorous extract completely.

Figure 5 shows the electronic absorption spectra of SWNTs obtained from the fluorous extract (after

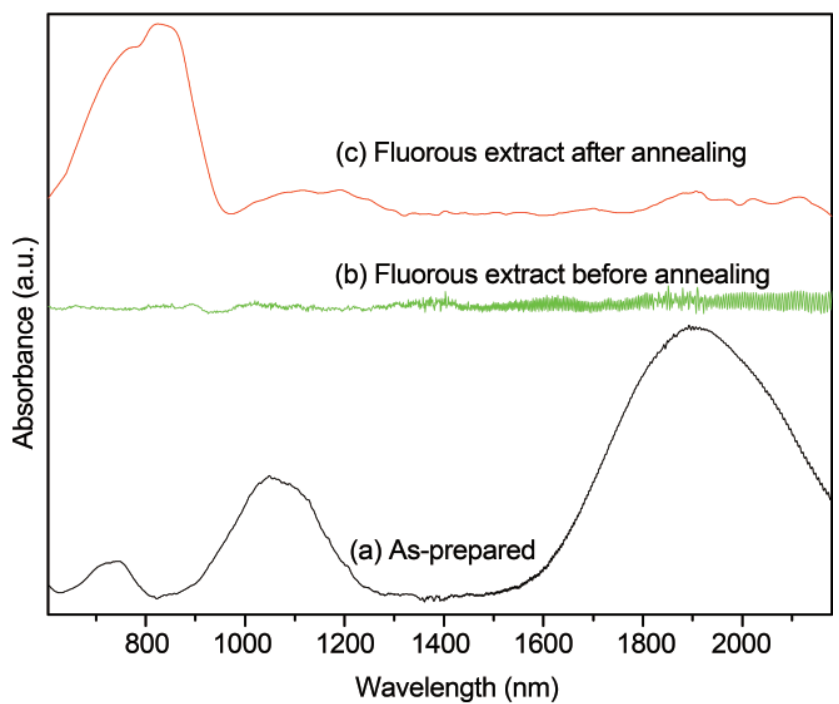

Figure 3 Electronic absorption spectra showing the evidence of defunctionalization of the metallic SWNTs: (a) as-prepared purified SWNTs, solid obtained from the fluorous extract (b) before, and (c) after removal of the diazonium functionality by heating heating it at $600{ }^{\circ} \mathrm{C}$ ) and the residue left over after the extraction, along with the spectrum of the asprepared sample. The residue shows almost no evidence of the metallic species in its absorption

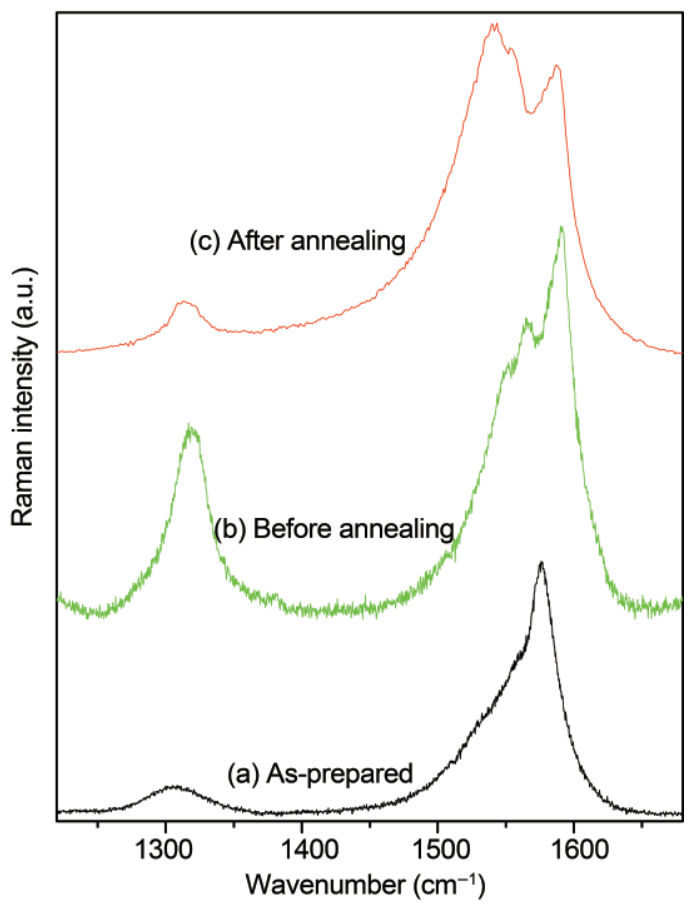

Figure 4 G- and D- bands in the Raman spectra (632 nm excitation laser) of the SWNT samples showing evidence of defunctionalization: (a) as-prepared pure SWNTs, solid obtained from the fluorous extract (b) before, and (c) after removal of the diazonium functionality by heating

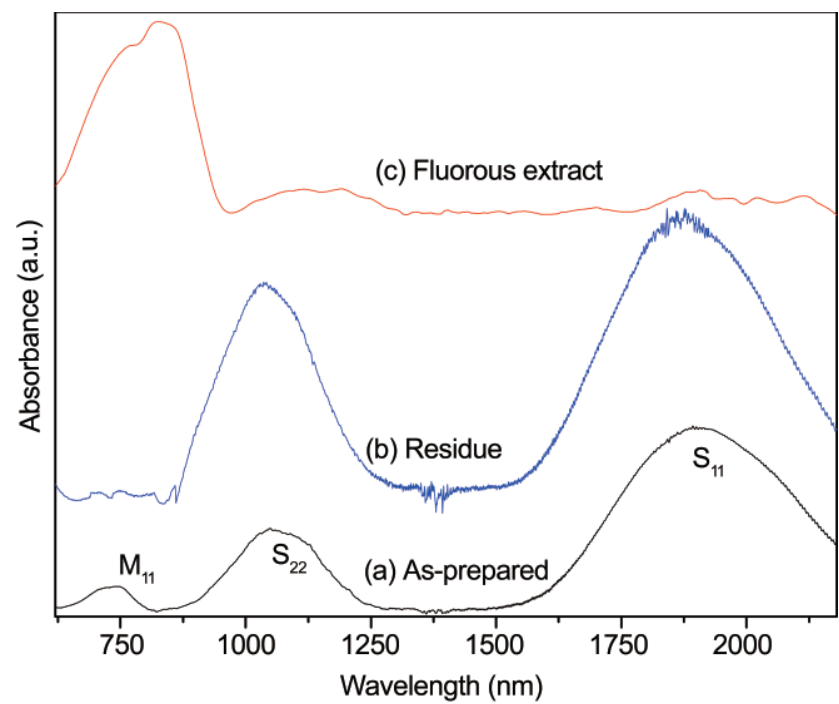

Figure 5 Electronic absorption spectra of the SWNT samples: (a) asprepared pure SWNTs; (b) residue left in the aqueous layer after the fluorous extraction and annealing at $600{ }^{\circ} \mathrm{C}$; (c) solid obtained from the fluorous extract after removal of the diazonium functionality by heating at $600{ }^{\circ} \mathrm{C}$ 
spectrum, but instead exhibits only the $S_{11}$ and $S_{22}$ bands. The solid obtained from the fluorous extract after the removal of the functionalization (after heating at $600{ }^{\circ} \mathrm{C}$ ) gives a spectrum which consists only of the metallic band $\mathrm{M}_{11}$ centered around $720 \mathrm{~nm}$. The absorbance data given are consistent with almost completely separated materials and not for mixtures slightly enriched by SWNTs of a particular electronic type. Even if the separated fractions contain some proportion of the other type of material (i.e., the fluorous fraction contains a very small amount of the semiconducting SWNTs or vice versa), the band intensities were so low that it was not possible to calculate the proportion of impurities in the separated metallic and semiconducting fractions. Thus, the electronic absorption spectra clearly demonstrate that the fluorous procedure is efficient in producing nearly pure fractions of semiconducting and metallic SWNTs.

The G-bands in the Raman spectra (recorded at $632 \mathrm{~nm}$ laser excitation) of the as-prepared residue and the fluorous extract are shown in Fig. 6. The spectra were recorded using solid samples obtained after washing with copious amounts of water to ensure that no other species were present along with the SWNTs. The spectrum of the as-prepared sample was decomposed into the BWF line shape [40] for the $1540 \mathrm{~cm}^{-1}$ band (the band due to metallic nanotubes) and Lorentzians for the 1555 and $1576 \mathrm{~cm}^{-1}$ bands (the bands due to semiconducting SWNTs). The G-band of the residue left after fluorous extraction shown in Fig. 6(b) clearly demonstrates a considerable reduction in the intensity of the $1540 \mathrm{~cm}^{-1}$ band showing that the sample consists almost entirely of semiconducting SWNTs. The $1540 \mathrm{~cm}^{-1}$ band shows a huge enhancement in the case of the fluorous extract (subjected to annealing at $600{ }^{\circ} \mathrm{C}$ ) showing that the sample comprises mostly metallic nanotubes as can be seen from Fig. 6(c). This demonstrates that the G-band of the fluorous extract is essentially due to the metallic species with their main characteristic band around $1540 \mathrm{~cm}^{-1}$. We have avoided calculating the percentage of metallic and semiconducting species since the Raman signal arises mainly from nanotubes with a narrow window of diameter and helicity that interact strongly with the photons. It is well known

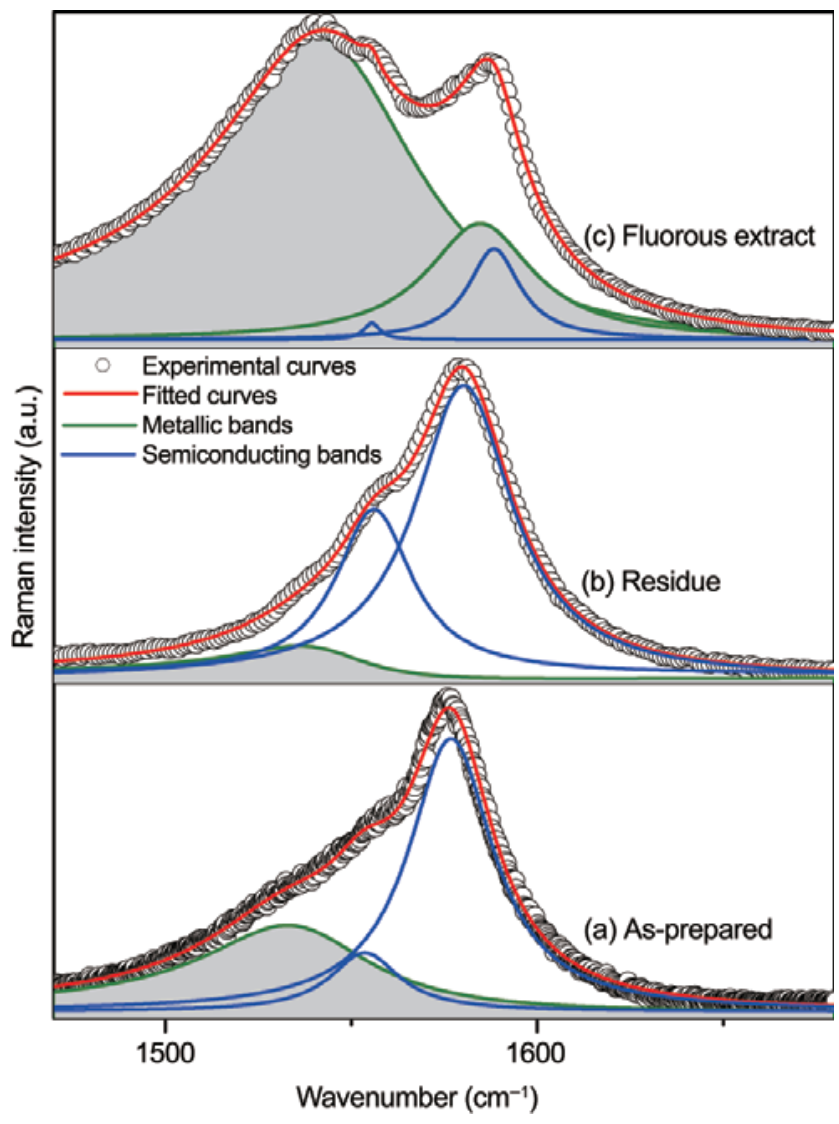

Figure 6 Decomposition of the G-bands in the Raman spectra (632 nm excitation laser) of the SWNT samples: (a) as-prepared pure SWNTs; (b) residue left in the aqueous layer after the fluorous extraction and annealing at $600{ }^{\circ} \mathrm{C}$; (c) solid obtained from the fluorous extract after removal of the diazonium functionality. The black circles represent the experimental spectrum and the fitted spectrum is shown by the red curve. The components of the G-bands due to semiconducting SWNTs are shown by the blue curves while the green curves with the area shaded below them represent the components due to the metallic SWNTs

that the appearance or disappearance of the BWF line shape depends on the presence of surfactants and other species [41] and the intensity of the BWF mode tends to be higher for surfactants containing electron donating groups, suggesting that charge injection may contribute to the BWF feature in dispersions of isolated SWNTs. To minimize these effects we ensured complete removal of the surfactant from the SWNTs by washing well with water before recording the spectra.

The RBM regions of the Raman spectra illustrated in Fig. 7 shows that the bands due to the semiconducting species have essentially disappeared in the spectrum of the fluorous extract while the 
bands due to the metallic species have disappeared in the case of the residue left after fluorous extraction.

We carried out $I-V$ measurements on the metallic (fluorous extract) and the semiconducting (residue) SWNTs obtained after the fluorous chemical procedure by employing scanning tunneling spectroscopy. We show typical results in Fig. 8 . We see the expected differences in the $I-V$ curves, with the band gap for the semiconducting species being $0.52 \mathrm{eV}$. The band gap of the semiconducting SWNT was calculated from the derivative of the I$V$ curve as shown in inset of the figure. Although we found semiconducting SWNTs with other band gap values, the value indicated here is the model value. The availability of pure semiconducting and metallic SWNTs has encouraged us to use them for investigations in sensor applications and also to investigate the effect of electron donor and acceptor molecules [42]. Electron donor molecules such as

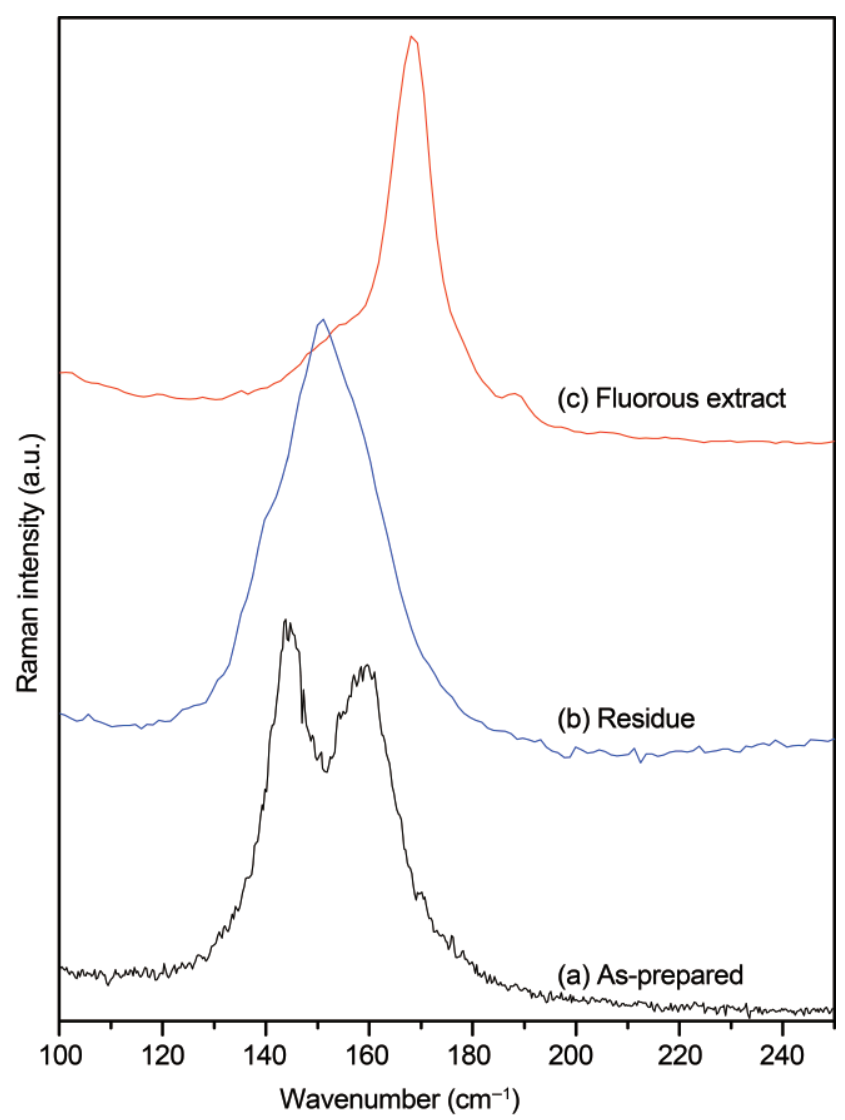

Figure 7 RBM bands in the Raman spectra of SWNTs: (a) asprepared pure SWNTs; (b) residue left in the aqueous layer after the fluorous extraction and annealing at $600{ }^{\circ} \mathrm{C}$; (c) solid obtained from the fluorous extract after removal of the diazonium functionality tetrathiafulvalene transform the semiconducting SWNTs into metallic ones while electron acceptors reduce the proportion of metallic nanotubes [43].

The separation of the SWNTs into metallic and semiconducting fractions is evident if one looks at the reaction mixture carefully before and after the reaction. As shown in Fig. 9, the suspension of the mixture of the SWNTs separates into two fractions, namely the fluorous and the aqueous fractions, after the chemical processing. When the aqueous reaction mixture was stirred with perfluorohexane overnight, frothing occurred because of the presence of the surfactant SDS. To remove the frothing so that the layers are visible to the eye, methanol was added to the solution which removed the SDS from the solution thereby yielding a clear interface. As the SDS was removed from the aqueous phase, the singly suspended SWNTs (which were suspended in solution by SDS) started aggregating as can be seen in

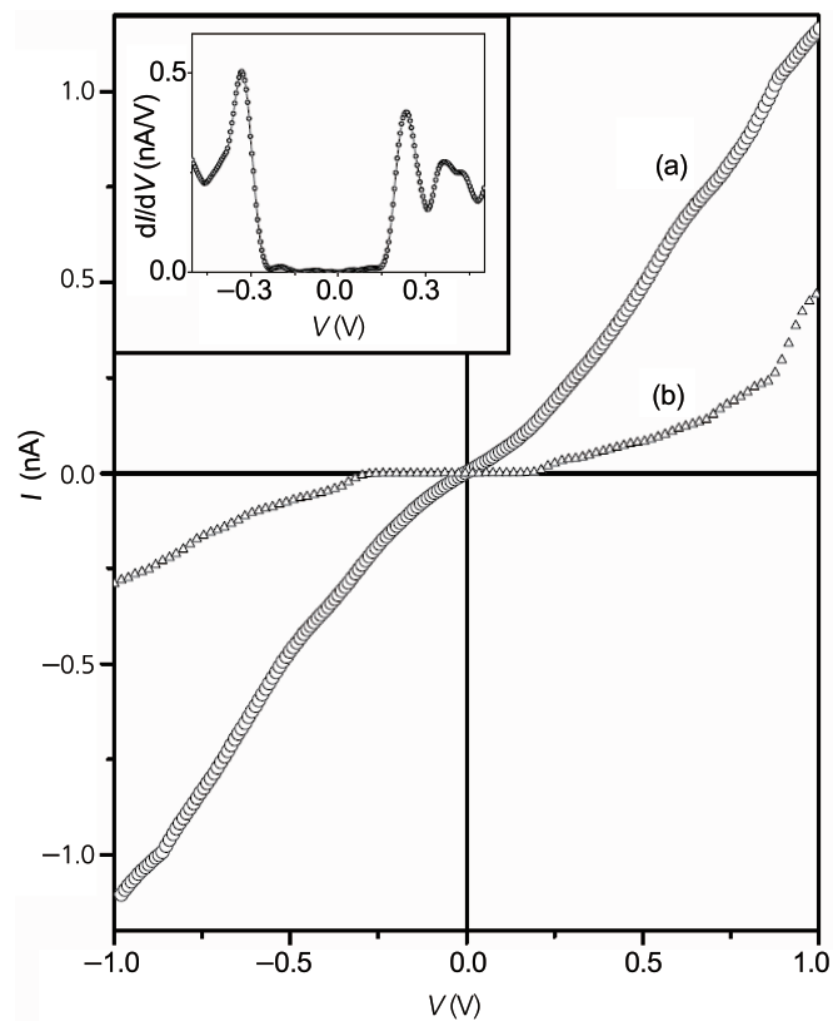

Figure $8 \quad I-V$ scanning tunneling spectroscopy measurements for separated SWNTs: (a) metallic SWNTs from the fluorous extract after the removal of the diazonium functionality; (b) semiconducting SWNTs from the aqueous residue. The inset shows the derivative of the $I-V$ curve obtained for the semiconducting SWNTs 


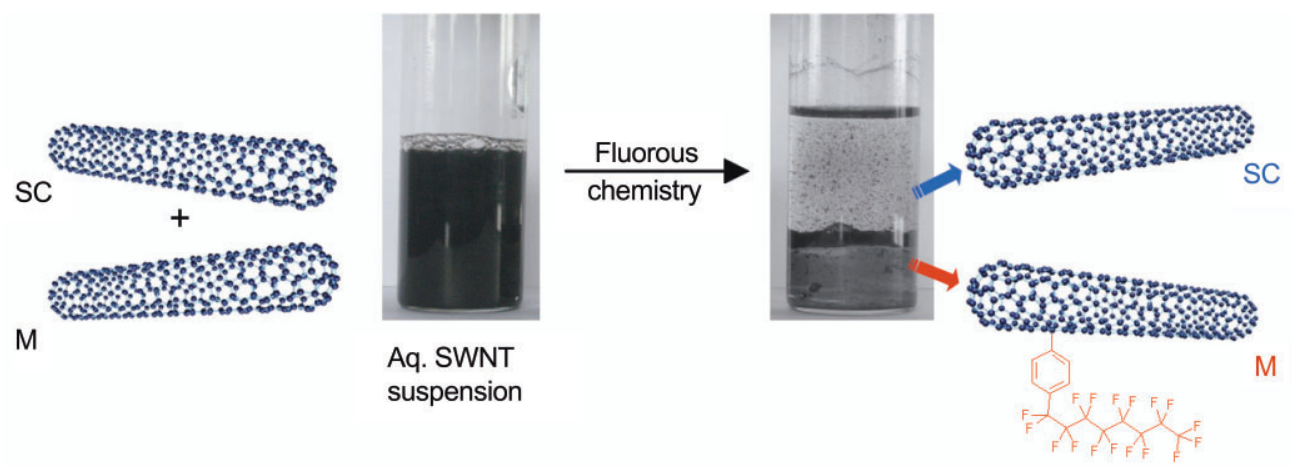

Figure 9 Reaction with a diazonium salt followed by fluorous extraction enables separation of semiconducting (upper aqueous layer) and metallic single walled carbon nanotubes (lower fluorous layer)

the top layer, whereas the bottom layer still showed a clear solution implying thereby that the fluorous tagged metallic SWNTs were being held in solution by the interaction between the perfluorohexane and the fluorous tag on the SWNTs.

In conclusion, by employing fluorous chemistry, we have been able to obtain nearly pure metallic and semiconducting SWNTs. The method is quite simple and avoids ultracentrifugation in the separation methodology. We used centrifugation only to produce singly suspended SWNTs in the presence of SDS. Using optical absorption data, we estimate that the metallic nanotubes obtained using the fluorous chemistry have a purity of at least $95 \%$. It should be possible to scale up the fluorous procedure and separate larger amounts of SWNTs into metallic and semiconducting nanotubes. Use of sonication and other such steps during the diazotization reaction may improve the efficacy of the procedure. The procedure based on fluorous chemistry can also be employed with SWNTs prepared by other methods such as HiPco and laser ablation.

\section{Acknowledgements}

The authors thank Ved Varun Agrawal for assistance with STM measurements.

\section{References}

[1] Saito, R.; Dresselhaus, G.; Dresselhaus, M. S. Physical Properties of Carbon Nanotubes; Imperial College Press: London, 1998.
[2] Rao, C. N. R.; Govindaraj, A. Nanotubes and Nanowires; RSC Publishing: Cambridge, 2005.

[3] Kukovecz, A.; Kramberger, C.; Georgakilas, V.; Prato, M.; Kuzmany, H. A. Detailed Raman study on thin single-wall carbon nanotubes prepared by the HiPCO process. Eur. Phys. J. B 2002, 28, 223-230.

[4] Appenzeller, J.; Martel, R.; Derycke, V.; Radosavljevic, M.; Wind, S.; Neumayer, D.; Avouris, P. Carbon nanotubes as potential building blocks for future nanoelectronics. Microelectron. Eng. 2002, 64, 391-397.

[5] Fan, S.; Chapline, M. G.; Franklin, N. R.; Tomber, T. W.; Cassell, A. M.; Dai, H. Self-oriented regular arrays of carbon nanotubes and their field emission properties. Science 1999, 283, 512-514.

[6] Kong, J.; Franklin, N. R.; Zhou, C.; Chapline, M. G.; Peng, S.; Cho, K.; Dai, H. Nanotube molecular wires as chemical sensors. Science 2000, 287, 622-625.

[7] Baughman, R. H.; Cui, C.; Zakhidov, A. A.; Iqbal, Z.; Barisci, J. N.; Spinks, G. M.; Wallace, G. G.; Mazoldi, A.; De Rossi, D.; Rinzler, A. G.; Jaschinski, O.; Roth, S.; Kertesz, M. Carbon nanotube actuators. Science 1999, 284, 1340-344.

[8] Ajayan, P. M. Aligned carbon nanotubes in a thin polymer film. Adv. Mater. 1995, 7, 489-491.

[9] Kamras, K.; Itkis, M. E.; Zhao, H.; Hu, B.; Haddon, R. C. Covalent bond formation to a carbon nanotube metal. Science 2003, 301, 1501.

[10] Strano, M. S.; Dyke, C. A.; Usrey, M. L.; Barone, P. W.; Allen, M. J.; Shan, H.; Kitrell, C.; Hauge, R. H.; Tour, J. M.; Smalley, R. E. Electronic structure control of single-walled carbon nanotube functionalization. Science 2003, 301, 1519-1522.

[11] Wang, Q.; Johnson, J. K. Optimization of carbon nanotube arrays for hydrogen adsorption. J. Phys. Chem. 
B 1999, 103, 4809-4813.

[12] Gülseren, O.; Ylidirim, T.; Ciraci, S. Effects of hydrogen adsorption on single-wall carbon nanotubes: Metallic hydrogen decoration. Phys. Rev. B 2002, 66, 121401.

[13] An, K. H.; Heo, J. G.; Jeon, K. G.; Bae, D. J.; Chulsu, J.; Yang, C. W.; Park, C. Y.; Lee, Y. H. X-ray photoemission spectroscopy study of fluorinated single-walled carbon nanotubes. Appl. Phys. Lett. 2002, 80, 42354237.

[14] Krupke, R.; Hennrich, F.; von Lohneysen, H.; Kappes, M. M. Separation of metallic from semiconducting singlewalled carbon nanotubes. Science 2003, 301, 344347.

[15] Chattopadhyay, D.; Galeska, L.; Papadimitrakopoulos, F. A route for bulk separation of semiconducting from metallic single-wall carbon nanotubes. J. Am. Chem. Soc. 2003, 125, 3370-3375.

[16] Li, H. P.; Zhou, B.; Lin, Y.; Gu, L.; Wang, W.; Fernando, K. A. S.; Kumar, S.; Allard, L. F.; Sun, Y. Selective interactions of porphyrins with semiconducting singlewalled carbon nanotubes. J. Am. Chem. Soc. 2004, 126, 1014-1015.

[17] Chen, Z.; Du, X.; Du, M.; Rancken, C.; Cheng, H.; Rinzler, A. Bulk separative enrichment in metallic or semiconducting single-walled carbon nanotubes. Nano Lett. 2003, 3, 1245-1249.

[18] Arnold, M. S.; Green, A. A.; Hulvat, J. F.; Stupp, S. I.; Hersam, M. C. Sorting carbon nanotubes by electronic structure using density differentiation. Nat. Nanotechnol. 2006, 1, 60-65.

[19] Toyoda, S.; Yamaguchi, Y.; Hiwatashi, M.; Tomonari, Y.; Murakami, H.; Nakashima, N. Separation of semiconducting single-walled carbon nanotubes by using a long-alkyl-chain benzenediazonium compound. Chem. Asian J. 2007, 2, 145-149.

[20] Zhang, W. Fluorous synthesis of heterocyclic systems. Chem. Rev. 2004, 104, 2531-2556.

[21] Yoshida, J.; Itami, K. Tag strategy for separation and recovery. Chem. Rev. 2002, 102, 3693-3716.

[22] Campidelli, S.; Menegheti, M.; Prato, M. Separation of metallic and semiconducting single-walled carbon nanotubes via covalent functionalization. Small 2007, 3 , 1672-1676

[23] Bahr, J. L.; Tour, J. M. Covalent chemistry of single-wall carbon nanotubes. J. Mater. Chem. 2002, 12, 19521958.
[24] Dyke, C. A.; Tour, J. M. Solvent-free functionalization of carbon nanotubes. J. Am. Chem. Soc. 2003, 125, 11561157.

[25] Bahr, J. L.; Yang, J.; Kosynkin, D. V.; Bronikowski, M. J.; Smalley, R. E.; Tour, J. M. Functionalization of carbon nanotubes by electrochemical reduction of aryl diazonium salts: A bucky paper electrode. J. Am. Chem. Soc. 2001, 123, 6536-6542.

[26] Kataura, H.; Kumazawa, Y.; Maniwa, Y.; Umezu, I.; Suzuki, S.; Ohtsuka, Y.; Achiba, Y. Optical properties of single-wall carbon nanotubes. Synth. Met. 1999, 103, 2555-2558.

[27] Rao, A. M.; Richter, E.; Bandow, S.; Chase, B.; Eklund, P. C.; Williams, K. A.; Fang, S.; Subbaswamy, K. R.; Menon, M.; Thess, A.; Smalley, R. E.; Dresselhaus, G.; Dresselhaus, M. S. Diameter-selective Raman scattering from vibrational modes in carbon nanotubes. Science 1997, 275, 187-191.

[28] Brown, S. D. M.; Corio, P.; Marucci, A.; Pimenta, M. A.; Dresselhaus, M. S.; Dresselhaus, G. Secondorder resonant Raman spectra of single-walled carbon nanotubes. Phys. Rev. B 2000, 61, 7734-7742.

[29] Brown, S. D. M.; Corio, P.; Marucci, A.; Dresselhaus, M. S.; Pimenta, M. A.; Kneipp, K. Anti-Stokes Raman spectra of single-walled carbon nanotubes. Phys. Rev. B 2000, 61, R5137-R5140.

[30] Das, A.; Sood, A. K.; Govindaraj, A.; Marco Saitta, A.; Lazzeri, M.; Mauri, F.; Rao, C. N. R. Doping in carbon nanotubes probed by Raman and transport measurements. Phys. Rev. Lett. 2007, 99, 136803.

[31] Scolari, M.; Mews, A.; Fu, N.; Myalitsin, A.; Assmus, T.; Balasubramanian, K.; Burghard, M.; Kern, K. Surface enhanced Raman scattering of carbon nanotubes decorated by individual fluorescent gold particles. J. Phys. Chem. C 2008, 112, 391-396.

[32] Vivekchand, S. R. C.; Govindaraj, A.; Sheikh M. M.; Rao C. N. R. New method of purification of carbon nanotubes based on hydrogen treatment. J. Phys. Chem. B 2004, 108, 6935-6937.

[33] O'Connell, M. J.; Bachilo, S. M.; Huffman, C. B.; Moore, V. C.; Strano, M. S.; Haroz, E. H.; Rialon, K. L.; Boul, P. J.; Noon, W. H.; Kittrell, C.; Ma, J.; Hauge, R. H.; Weisman, R. B.; Smalley, R. E. Band gap fluorescence from individual single-walled carbon nanotubes. Science 2002, 297, 593-596.

[34] Yoshino, N.; Kitamura, M.; Seto, T.; Shibata, Y.; Abe, 
M.; Ogino, K. Synthesis of azobenzene derivatives having fluoroalkyl chain and their monomolecular film formation at the air/water interface. Bull. Chem. Soc. Jpn. 1992, 65, 2141-2144.

[35] Fantini, C.; Jorio, A.; Souza, M.; Strano, M. S.; Dresselhaus, M. S.; Pimenta, M. A. Optical transition energies for carbon nanotubes from resonant Raman spectroscopy: Environment and temperature effects. Phys. Rev. Lett. 2004, 93, 147406.

[36] Anderson, N.; Hartschuh, A.; Cronin, S.; Novotny, L. Nanoscale vibrational analysis of single-walled carbon nanotubes. J. Am. Chem. Soc. 2005, 127, 25332537.

[37] Maeda, Y.; Takano, Y.; Sagara, A.; Hashimoto, M.; Kanda, M.; Kimura, S.; Lian, Y.; Nakahodo, T.; Tsuchiya, T.; Wakahara, T.; Akasaka, T.; Hasegawa, T.; Kazaoui, S.; Minami, N.; Lu, J.; Nagase, S. Simple purification and selective enrichment of metallic SWCNTs produced using the arc-discharge method. Carbon 2008, 46, 15631569.

[38] Zhu, H. -W.; Jiang, B.; Xu, C. -L; Wu, D. -H. Synthesis of high quality single-walled carbon nanotube silks by the arc discharge technique. J. Phys. Chem. B 2003, 107, 6514-6518.

[39] Tarasov, B. P.; Muradyan, V. E.; Shul'ga, Y. M.;
Krinichnaya, E. P.; Kuyunko, N. S.; Efimov, O. N.; Obraztsova, E. D.; Schur, D. V.; Maehlen, J. P.; Yartys, V. A.; Lai, H. -J. Synthesis of carbon nanostructures by arc evaporation of graphite rods with $\mathrm{Co}-\mathrm{Ni}$ and $\mathrm{YNi}_{2}$ catalysts. Carbon 2003, 41, 1357-1364.

[40] Brown, S. D. M.; Jorio, A.; Corio, P.; Dresselhaus, M. S.; Dresselhaus, G.; Saito, R.; Kneipp, K. Origin of the Breit-Wigner-Fano lineshape of the tangential G-band feature of metallic carbon nanotubes. Phys. Rev. B 2001, $63,155414$.

[41] Blackburn, J. L.; Engtrakul, C.; McDonald, T. J.; Dillon, A. C.; Heben, M. J. Effects of surfactant and boron doping on the BWF feature in the Raman spectrum of singlewall carbon nanotube aqueous dispersions. J. Phys. Chem. B 2006, 110, 25551-25558.

[42] Shin, H. -J.; Kim, S. M.; Yoon, S. -M.; Benayad, A.; Kim, K. K.; Kim, S. J.; Park, H. K.; Choi, J. -Y.; Lee, Y. H. Tailoring electronic structures of carbon nanotubes by solvent with electron-donating and -withdrawing groups. J. Am. Chem. Soc. 2008, 130, 2062-2066.

[43] Voggu, R.; Rout, C. S.; Franklin, A. D.; Fisher, J. S.; Rao, C. N. R. Extraordinary sensitivity of the electronic structure and properties of single-walled carbon nanotubes to molecular charge-transfer. J. Phys. Chem. C 2008, 112, 13053-13056. 